\title{
Monotonicity Preserving SIRMs-Connected Fuzzy Inference Systems with a New Monotonicity Index: Learning and Tuning
}

\author{
${ }^{1}$ See Hung Lau, ${ }^{2}$ Kai Meng Tay, ${ }^{3}$ Chee Khoon Ng \\ Faculty of Engineering, Universiti Malaysia Sarawak, Malaysia \\ ${ }^{1}$ lauseehung@gmail.com, ${ }^{2}$ kmtay@feng.unimas.my, ${ }^{3}$ ckng@feng.unimas.my
}

\begin{abstract}
Recent research on Single Input Rule Modules (SIRMs)-connected fuzzy inference system (FIS) focuses on its monotonicity property fulfillment. The aim of this paper is to propose an alternative approach for modeling of monotonicitypreserving SIRMs-connected FIS. A new monotonicity index (MI) for approximating the monotonicity property fulfillment of an SIRMs-connected FIS is proposed. A hybrid of Harmony Search (HS), SIRMs-connected FIS, and the new MI is investigated. A proposed data-driven monotonicity-preserving SIRMs-connected FIS model with HS is then presented. The use of MI for tuning of an SIRMs-connected FIS is demonstrated too.
\end{abstract}

Keywords-single input rule modules connected fuzzy inference system, harmony search, monotonicity index, data-driven, learning, tuning

\section{INTRODUCTION}

Recent advances in fuzzy modeling focus on optimizationbased fuzzy inference systems (FIS) [1-5] and the fulfillment of theoretical properties for various FIS models [6-10]. The former focuses on the use of an optimization tool (e.g., genetic algorithm, particle swarm optimization and etc.) for designing an FIS model by providing them with learning and/or tuning capabilities. Herrera [5] proposed a taxonomy for genetic fuzzy systems (GFSs) and two important types of GFSs are genetic learning and genetic tuning. On one hand, genetic learning focuses on learning of FIS from numerical data. On the other hand, genetic tuning attempts to improve the performance of an FIS by adjusting some parameters, usually membership function only.

The latter focuses on the investigation of theoretical properties of various FIS models, e.g., monotonicity [6-11], robustness [12], and continuity [13]. The importance of monotonicity property has been highlighted in a number of recent publications [6-9], [11], [14-17]. From the literature, the mathematical conditions of various FIS models (e.g., Mamdani FIS [6], [14], Sugeno FIS [7], [17], and SIRMs-connected FIS [9-10], [18]) has been developed and adopted as governing equations in an FIS modeling process [6-7], [9], [11], [18-19] to observe the monotonicity property. It is worth mentioning that in [20], a new fuzzy failure mode and effect analysis (FMEA) methodology with SIRMs-connected FIS was proposed, and a theorem from [9-10], [18] are adopted as governing equations.

Another alternative approach for the study of monotonicity property is perhaps the development of a monotonicity index (MI) or test procedure to approximate or estimate the monotonicity fulfillment. In our previous works [15-16], [21], MI has been developed and applied to FIS with learning, and similarity reasoning features. The aim of this paper is to propose a new MI for SIRMs-connected FIS. In this paper, MI is used instead of mathematical conditions from [9-10], [18], for the reasons as follow; (1) the developed mathematical constraints are a set of sufficient conditions, viz. a SIRMsconnected FIS may be of the monotonicity property even if the mathematical constraints are not satisfied, and (2) the inclusion of the mathematical constraints in optimization-based FIS models may complicate the search process. It is however worth mentioning that MI is an approximate approach, instead of an analytical approach.

We further show the applicability of the proposed MI for optimization-based FIS models, i.e., FIS with learning and FIS with tuning feature. In this paper, HS is adopted, for the reasons as follow; (1) it preserves the history of past vectors (similar to Tabu Search (TS)); (2) it allows the adaption rate to be varied from the beginning to the end; (3) it manages several vectors simultaneously in a manner similar to genetic algorithm (GA); and (4) HS considers each component variable in a vector independently while it generates a new vector in which GA cannot perform [22].

The HS-based SIRMs-connected FIS with learning abilities attempts to search for an SIRMs-connected FIS which best fit a set of data (i.e., with the minimal error), while satisfying the monotonicity property fulfillment. It is worth mentioning that in [10], monotonicity property and learning feature for SIRMsconnected FIS have been studied. However, it is not clear, how to develop a monotonicity-preserving SIRMs-connected FIS model with learning feature. Our proposed approach is a solution to this research gap. The HS-based SIRMs-connected FIS with tuning ability search for a monotone SIRMsconnected FIS which is "nearest" to a non-monotone SIRMsconnected FIS.

In this paper, two implementations of MI are studied, i.e., static and dynamic. The static approach assumes that the parameters are fixed and static in the HS searching process. The dynamic approach attempts to vary these search parameters in the HS process, i.e., different (random) set of parameters in every iteration.

This paper is organized as follows. In section II, SIRMsconnected FIS model and HS algorithm are reviewed. In section III, MIs for SIRMs-connected FIS is developed. A general formulation for optimization-based SIRMs-connected FIS is also presented. In section IV, a monotonicity-preserving SIRMs-connected FIS with learning feature is presented and 